\title{
La proopiomélanocortine et ses fragments : application à I'investigation clinique
}

La proopiomélanocortine (POMC) est le précurseur normal de l'ACTH et d'autres peptides dont l'action biologique est mal connue. La maturation protéolytique du précurseur en ses dérivés finaux varie selon les tissus producteurs, lobes antérieur et intermédiaire de l'hypophyse, tumeurs à sécrétion ectopique. De ce fait, le dosage (par des méthodes immunologiques éventuellement associées à l'HPLC) des différents fragments de la POMC peut orienter sur l'origine d'un hypercorticisme et permet de suivre l'évolution sous traitement de tumeurs avec sécrétion inappropriée d'ACTH.

\section{Didier Vieau Christine Gicquel Marie-Françoise Proeschel}

\section{ADRESSES}

D. Vieau : docteur es sciences. G. Gicquel : docteur en médecine, ancien interne des hôpitaux de Paris. M.-F. Proeschel : technicienne. Centre de recherche sur les maladies endocriniennes, hôpital Cochin. Laboratoire d'explorations fonctionnelles, hôpital Trousseau, 26, avenue du docteur Arnold Net- a proopiomélanocortine (POMC) est le précurseur polypeptidique de l'ACTH. Bien que le gène $P O M C$ s'exprime en réalité dans de nombreux tissus sains, il est admis que l'ACTH circulante chez l'homme provient en totalité de la seule sécrétion antéhypophysaire par les cellules corticotropes. Quelques rares tumeurs non hypophysaires sont occasionnellement la source d'une sécrétion inappropriée et excessive d'ACTH créant un syndrome de Cushing paranéoplasique: ce type d'affection, décrit au début des années 1960 par le groupe de Liddle [1], fait de l'ACTH le prototype des sécrétions dites ectopiques. On sait aujourd'hui que ce phénomène s'applique à d'autres hormones et qu'il n'est pas toujours si ectopique que cela!

Les mécanismes moléculaires qui aboutissent à la synthèse d'ACTH sont aujourd'hui parfaitement connus. Ils nous apprennent que l'ACTH n'est qu'un morceau - sans doute le plus important - d'un puzzle qui compte de nombreuses autres pièces. Le clinicien ou l'investigateur qui souhaite manipuler ou évaluer la fonction corticotrope ne peut ignorer cette nouvelle donne. Son apparente complexité offre en fait de nouvelles cibles pour explorer de façon encore plus performante la fonction corticotrope saine ou pathologique.

\section{La POMC : rappel historique}

Abe et al., en 1969 [2], observent que $\mathrm{ACTH}$ et " $\beta$-MSH " varient toujours de façon parallèle dans le sang. En 1971, Yalow et Berson [3] mettent en évidence dans une tumeur thymique humaine une molécule possédant une immunoréactivité ACTH mais ayant un poids moléculaire beaucoup plus élevé, d'environ 22 000. Cette molécule, inactive, libère, sous l'action ménagée de la trypsine, l'ACTH ${ }_{1.39}$ biologiquement active. En 1974, Bloomfield et al. [4] montrent que la $\beta$-MSH circulante est en fait une lipotropine (LPH) ; la même année, Phifer et al. [5] retrou- 
vent par immunohistochimie l'ACTH et les LPH dans la même cellule antéhypophysaire. En 1977, les expériences de pulse-chase (marquage isotopique suivi de chasse avec du substrat non radiomarqué) menées par Mains et al. [6] sur les cellules de la lignée tumorale corticotrope de souris AtT20/D16v permettent une approche plus dynamique de la maturation de la POMC, démontrant pour la première fois l'existence d'un précurseur commun à l'ACTH et à la $\beta$-LPH dans ce modèle expérimental. Un tel précurseur sera ensuite identifié chez l'homme dans une lignée dérivée d'un cancer bronchique anaplasique à petites cellules [7].

La séquence de ce précurseur sera définitivement établie grâce à la génétique moléculaire. Le groupe de Numa isole, à partir du lobe intermédiaire de l'hypophyse de bœuf, un ARN messager (ARNm) dont le produit de traduction est précipité par les antisérums anti-ACTH. Le même groupe clone l'ADN complémentaire $(\mathrm{ADN})$ de ce messager et détermine sa séquence nucléotidique à partir de laquelle est déduite la structure primaire de la POMC bovine [8]. L'ADNc obtenu à partir de la lignée AtT20 révèle une forte homologie de séquence entre les précurseurs de ces deux espèces. Les séquences des $\mathrm{ADNc}$ et l'organisation génique du précurseur seront par la suite établies dans de nombreuses espèces, dont l'homme [9, 10]. Les mécanismes moléculaires de la synthèse d'ACTH sont donc entièrement dévoilés ; ainsi se trouve résolu le puzzle moléculaire qui permet de réunir ACTH, LPH, $\beta$-endorphine...

\section{Le système POMC humain : description}

\section{- Le gène}

Le gène de la POMC, unique chez l'homme, est situé sur le bras court du chromosome 2 dans la bande p23. Il comporte 7665 paires de bases (pb) et est composé de 3 exons et de 2 introns ((figure 1).

- L'exon 1 est constitué de 87 pb et correspond à la partie 5' non traduite du messager.

- L'exon 2 est composé de 152 pb et comprend une partie non traduite ainsi que les nucléotides codant pour $m / s n^{\circ} 9$, vol. 7. nourembre 91

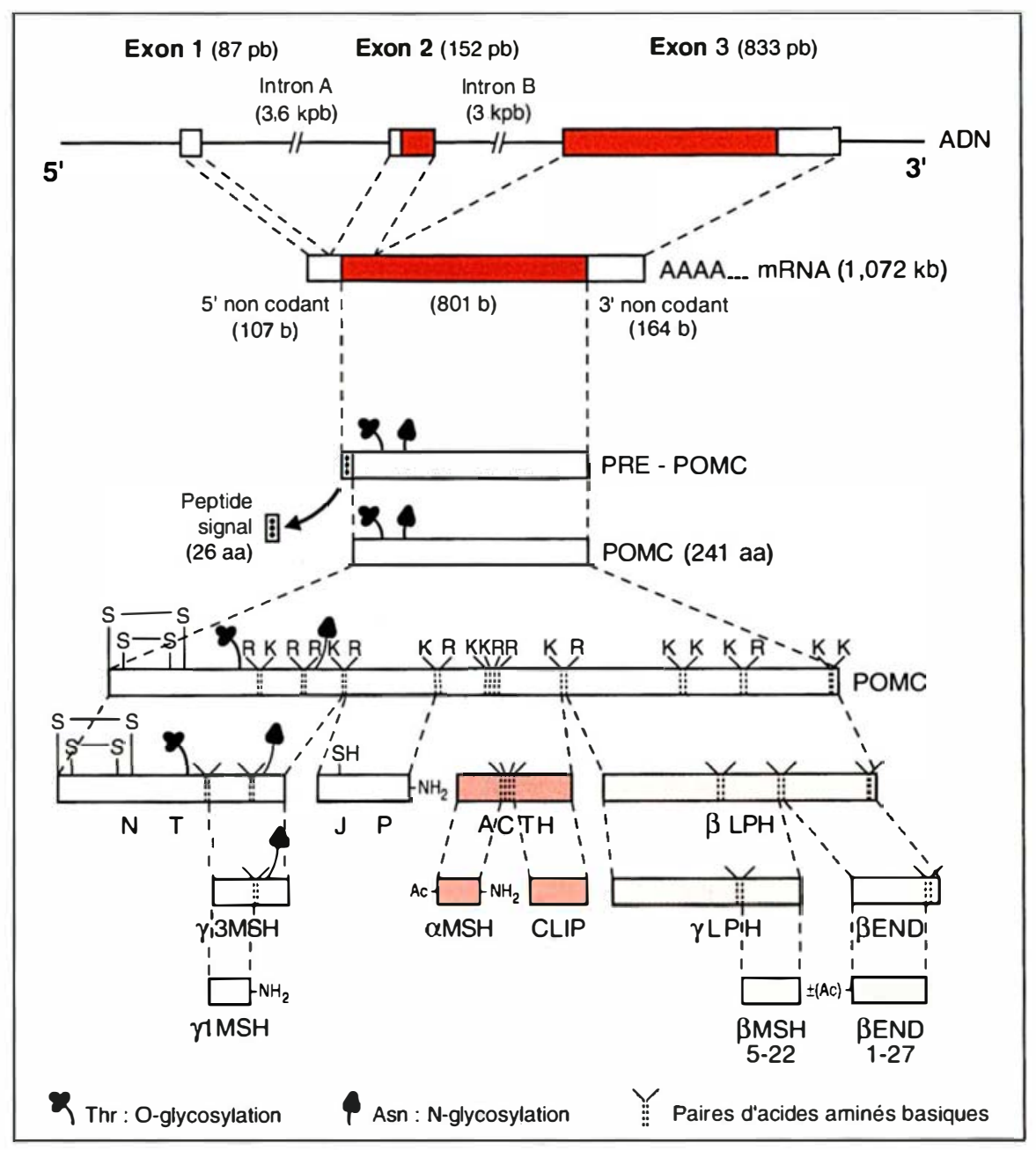

Figure 1. Vue schématique de l'expression du gène POMC humain. Les parties codantes de I'ADN et de l'ARN sont représentées en rouge. Les peptides retrouvés dans le lobe antérieur de l'hypophyse humaine sont schématisés par des rectangles hachurés. NT : fragment $N$-terminal ; JP : joining peptide ; $A T C H$ : adrenocorticotropin hormone ; $L P H$ : lipotropin hormone ; $M S H$ : melanostimulin hormone ; END : endorphine ; CLIP : corticotropin-like intermediary lobe peptide.

le peptide signal et les premiers acides aminés du fragment N-terminal (NT) de la POMC.

- L'exon 3 est constitué de 833 pb et correspond à la partie du gène codant pour pratiquement tout le précurseur [partie C-terminale (C-ter) du NT, joining peptide (JP), ACTH et $\beta$-LPH] ainsi qu'à la partie 3' non traduite du messager.

Les deux longues séquences introniques $\mathrm{A}$ et $\mathrm{B}$, respectivement de 3708 et $2886 \mathrm{pb}$, présentent des séquences répétitives de la famille Alu. La comparaison des séquences nucléotidiques de différentes espèces a montré une forte homologie au niveau des trois régions codant pour le $\mathrm{NT}$, l'ACTH, la $\beta-\mathrm{MSH}$ et la $\beta$ endorphine alors que les régions codant pour le JP et la $\gamma$ LPH divergent beaucoup selon les espèces.

- Expression du gène POMC dans l'hypophyse normale

Le gène de la POMC humaine transcrit dans les cellules corticotropes antéhypophysaires engendre après maturation des ARN nucléaires précurseurs un ARNm de 1072 nucléotides dont 107 non codants à l'extrémité 5 ' et 164 non codants à l'extrémité 3'. Un épissage alternatif très minoritaire (environ $5 \%$ ) ajoute 


\section{RÉFÉRENCES}

1. Liddle GW, Nicholson WE, Island DP, Orth DN, Abe K, Lowder SC. Clinical and laboratory studies of ectopic humoral syndromes. Rec Prog Horm Res 1969; 25 : 283-314.

2. Abe K, Nicholson WE, Liddle GW, Orth DN, Island DP. Normal and abnormal regulation of $\beta-\mathrm{MSH}$ in man. J Clin Invest $1969 ; 45: 1580-5$.

3. Yalow RS, Berson SA. Size heterogeneity of immunoreactive human ACTH in plasma and in extracts of pituitary glands and ACTH-producing thymoma. Biochem Biophys Res Commun 1971; 44 : 439-45.

4. Bloomfield GA, Scott AP, Lowry PJ Gilkes JJH, Rees LH. A reappraisal of human $\beta$-MSH. Nature $1974 ; 252: 492-3$.

5. Phifer RF, Orth DN, Spicer SS. Specific demonstration of the human hypophy. seal adrenocorticomelanotropic (ACTH/ MSH) cell. J Clin Endocrinol Metab 1974 $39: 684-92$.

6. Mains RE, Eipper BA, Ling N. Common precursor to corticotropins and endorphins. Proc Natl Acad Sci USA 1977; 74 3014-8.

7. Bertagna $X$, Nicholson WE, Sorenson GD, Pettengill OS, Mount CD, Orth DN Corticotropin, lipotropin and $\beta$-endorphin production by a human nonpituitary tumor in tissue culture : evidence for a common precursor. Proc Natl Acad Sci USA 1978 $75: 5160-4$

8. Nakanishi $\mathrm{S}$, Anoue $\mathrm{A}$, Kita $\mathrm{T}$, et al. Nucleotide sequence of cloned cDNA for bovine corticotropin- $\beta$-lipotropin precursor. Nature 1979 ; 278 : 423-7.

9. Whitfeld P, Seeburg P, Shine J. The human proopiomelanocortin gene : organization, sequence and interspersion with repetitive DNA. DNA $1982 ; 1: 133-43$.

10. Takahashi H, Hakamata Y. Watanabe Y, Kikuno R, Miyata T, Numa S. Complete nucleotide sequence of the human corticotropin- $\beta$-lipotropin precursor gene. Nucleic Acids Res 1983 ; 11: 6847-8.

11. De Keyzer Y, Bertagna X, Luton JP, Kahn A. Variable modes of proopiomelanocortin gene transcription in human tumors. Mol Endocrinol $1989 ; 3: 215-23$

\footnotetext{
* Voir aussi article de D. Germain et al., p. 895 de ce numéro.

** Un premier anticorps en large excès fixe la molécule recherchée par une extrémité, un second anticorps égalament en excès et marqué se lie ensuite à cette mâme molécule par son autre extrémité. La quantité de radioactivité retrouvée associée au premier anticorps (souvent fixé sur une phose solide) est directement proportionnelle à la quantité de molécules recherchées. La "prise en sandwich " de la molecule par les doux anticorps confère à l'IRMA sa grande spécificité de reconnaissance.
}

30 nucléotides à la partie 5' du deuxième exon, sans conséquence sur le cadre de lecture d'une partie non traduite du messager [11]. Les 801 nucléotides de la région codante sont traduits par les ribosomes du réticulum endoplasmique rugueux et donnent naissance à une protéine de 267 acides aminés portant à son extrémité $\mathrm{N}$-ter un peptide signal hydrophobe de 26 acides aminés. Au cours de la traduction, ce fragment peptidique est reconnu par un complexe ribonucléoprotéique (SRP ou signal recognition particle). Un récepteur spécifique du SRP ancre la protéine naissante dans la membrane du réticulum et guide sa translocation dans la lumière de celui-ci. Le peptide signal est alors rapidement clivé avant même que la protéine ait quitté le ribosome. La protéine ainsi guidée pénètre entièrement dans le réticulum et est engagée dans le transit intracellulaire propre aux protéines destinées à être sécrétées ou incorporées dans une membrane ainsi qu'aux enzymes lysosomiales. Après coupure du peptide signal la POMC est constituée par la juxtaposition des peptides suivants : le NT, le JP, l'ACTH et la $\beta$-LPH dont les 31 acides aminés de l'extrémité C-ter constituent la $\beta$-endorphine.

- Expression du gène POMC dans les tissus non hypophysaires normaux

De nombreux auteurs ont confirmé la présence d'ARNm POMC dans les tissus non hypophysaires, chez l'animal comme chez l'homme [12-14]. Dans la plupart de ces tissus (gonades, thymus, poumons...), le mode d'expression du gène POMC est quantitativement et qualitativement différent : les niveaux d'ARN messager sont beaucoup plus faibles que dans l'hypophyse ; surtout, on détecte essentiellement un messager court, tronqué, ne possédant qu'environ 800 nucléotides. Ces messagers résultent d'un phénomène de transcription démarrant de façon hétérogène en plusieurs points situés dans la partie 5' du troisième exon. Ces messagers, selon toute vraisemblance, ne peuvent être traduits en peptides de type POMC.

Dans le cerveau, toutefois, certains neurones hypothalamiques expriment de façon majoritaire un messager de type hypophysaire responsable de la production locale de POMC, précurseur de la $\beta$-endorphine cérébrale [15].

\section{- Maturation de la POMC}

Une fois introduite dans le réticulum endoplasmique la POMC va transiter par l'appareil de Golgi à partir duquel se forment les granules de sécrétion où les produits de maturation sont stockés avant d'être sécrétés par exocytose. Au cours de ce transit, la POMC subit, en effet, un ensemble de modifications sous l'effet de clivages protéolytiques et de modifications chimiques réunies sous le terme de maturation [16].

\section{- Maturation protéolytique}

Plusieurs clivages protéolytiques de type trypsique libèrent une série de peptides, dont l'ACTH. Les enzymes impliquées ont une spécificité connue depuis longtemps. Elles utilisent des sites pratiquement toujours constitués de paires d'acides aminés basiques arginine $(\mathrm{R})$ et lysine $(\mathrm{K})$ dans un ordre quelconque. Ce phénomène n'est d'ailleurs pas limité à la POMC mais s'applique à tous les précurseurs polypeptidiques. Il existe neuf sites de ce type sur la POMC (figure 1): (1) dans le lobe antérieur 4 seulement de ces sites sont utilisés, tous de type lysine-arginine. Cinq peptides sont produits le NT, le JP, l'ACTH, la $\beta$-LPH ainsi qu'un peu de $\gamma$-LPH et de $\beta$ endorphine, ce dernier site n'étant que partiellement utilisé ; (2) dans le lobe intermédiaire, vestigial chez l'homme, tous les sites sont utilisés aboutissant à une série de peptides plus courts ; le NT donne naissance aux $\gamma-\mathrm{MSH}$, l'ACTH à l' $\alpha$-MSH et au corticotropin-like intermediary lobe peptide (CLIP ou ACTH ${ }_{18.39}$ ), la $\beta$-LPH est entièrement clivé en $\beta$-endorphine et en $\beta$-MSH. De plus, la $\beta$-endorphine existe sous différentes formes plus ou moins raccourcies, $\beta$-endorphine ${ }_{1.27}$ ou ${ }_{1-26}$.

Restées longtemps énigmatiques, ces enzymes ou prohormones convertases viennent d'être identifées [17, 18]. Le groupe de Chrétien, à Montréal, a cloné les deux enzymes PC1 et PC2 et montré comment elles étaient impliquées dans la maturation différentielle de la POMC dans les lobes antérieur et intermédiaire de l'hypophyse [19, 20]*. 


\section{- Les modifications chimiques}

Ces fragments peptidiques subissent encore des modifications chimiques telles que glycosylation, amidation, phosphorylation et acétylation.

- Le fragment NT subit une Nglycosylation sur l'acide aminé Asn ${ }^{45}$ et une O-glycosylation sur l'acide aminé $\mathrm{Thr}^{45}$. Ces modifications conferent à ce peptide de 76 acides aminés un poids moléculaire d'environ 11000 [21].

- L'ACTH est en partie phosphorylée sur la sérine ${ }^{31}$ par une enzyme caséine kinase de type 2 [22]. - Le JP est amidé sous la forme d'un peptide de 30 acides aminés après clivage de la glycine C-ter [23] (l'article de Ouafik, dans le même numéro, décrit dans le détail les mécanismes de cette amidation).

- Dans le lobe intermédiaire, enfin, les mêmes modifications du JP et du fragment résiduel du NT sont observées; la $\beta$-endorphine et l' $\alpha$-MSH sont de plus acétylées à leur extrémité $\mathrm{N}$-ter, l' $\alpha$-MSH est amidée à son extrémité C-ter [24].

Ces modifications chimiques sont donc une source supplémentaire de diversité des peptides dérivés de la POMC et certaines d'entre elles sont étroitement associées au type de la cellule productrice. Dans certains cas, de plus, elles peuvent avoir un retentissement sur l'activité biologique du peptide concerné. L'acétylation supprime ainsi totalement l'activité morphinomimétique de la $\beta$-endorphine alors qu'elle accroît l'activité mélanostimulante de l' $\alpha$-MSH $[25,26]$.

\section{Effets biologiques des peptides circulants}

Le phénomène de maturation posttraductionnelle implique qu'au cours de l'exocytose l'ensemble des peptides de la POMC contenus dans le même granule de sécrétion soit libéré de façon simultanée dans la circulation sanguine. En dehors de l'ACTH, le rôle physiologique des peptides de la POMC n'est pas clairement identifié; pour certains d'entre eux, cependant, une activité biologique a été décrite.

Pedersen et Brownie [27] ainsi que Seidah et al. [28] ont montré que le NT stimulait la production d'aldostérone par une action synergique avec
l'ACTH. Un rôle trophique sur la corticosurrénale a également été invoqué pour la $\gamma 3 \mathrm{MSH}$ et d'autres peptides dérivés de la région $\mathrm{N}$-ter [29].

Aucune action du JP n'est connue à l'heure actuelle. Il avait été proposé que le $\mathrm{JP}_{1-12}$ soit un stimulant spécifique des androgènes de la corticosurrénale, mais cette hypothèse a été infirmée récemment [30]. L'existence d'un tel peptide est d'ailleurs peu vraisemblable.

Les MSH $\alpha$ et $\beta$ ont une action mélanostimulante, de même que les $\mathrm{LPH}$, probablement due à leur séquence heptapeptidique commune $\left(\alpha-\mathrm{MSH}_{4-10}\right)$.

La $\beta$-endorphine est un des plus puissants peptides endogènes à action opiacée. Il est à remarquer que la $\beta$ endorphine contient une séquence identique à la met-enképhaline, mais cette séquence n'est pas encadrée de paires d'acides aminés basiques à l'intérieur de la POMC et elle n'est donc pas libérée par ce précurseur. La $\beta$-endorphine ne franchit pas la barrière hémato-encéphalique et, par conséquent, la forme circulante sécrétée par l'hypophyse ne peut intervenir à ce niveau. Quant à la $\beta$-endorphine sécrétée par le lobe intermédiaire, elle est acétylée, ce qui la rend incapable de se lier aux récepteurs opiacés.

\section{Évaluation de la fonction corticotrope}

\section{- L'ACTH : rappel historique}

La première approche clinique fut effectuée en 1961 par le groupe de Liddle en utilisant le dosage biologique de Lipscomb et Nelson [31]. Le premier dosage radio-immunologique (RIA) applicable à l' ACTH plasmatique apparut en 1964 [32]. Jusqu'à très récemment, le RIA fut la méthode de choix pour l'évaluation de la fonction corticotrope.

Depuis quelques années, plusieurs groupes ont développé et commercialisé des dosages immunoradiométriques (IRMA**) de l'ACTH [33]. Ces derniers dosages présentent des avantages par rapport aux RIA classiques en raison d'une spécificité absolue, puisque seule l'ACTH ${ }_{1-39}$ (qu'elle soit phosphorylée ou non) est reconnue. Bien que les fragments d'ACTH ne soient pas mesurés par cette méthode, ils peuvent, à fortes concentrations, saturer le premier anticorps et interférer dans le dosage d'ACTH ${ }_{1-39}$. L'IRMA présente d'autres intérêts : celui d'un dosage plasmatique direct ainsi que la rapidité d'exécution (moins de 24 heures). Il est dorénavant la méthode de choix pour la mesure de l'ACTH plasmatique dans les années à venir.

D'autres moyens ont également été

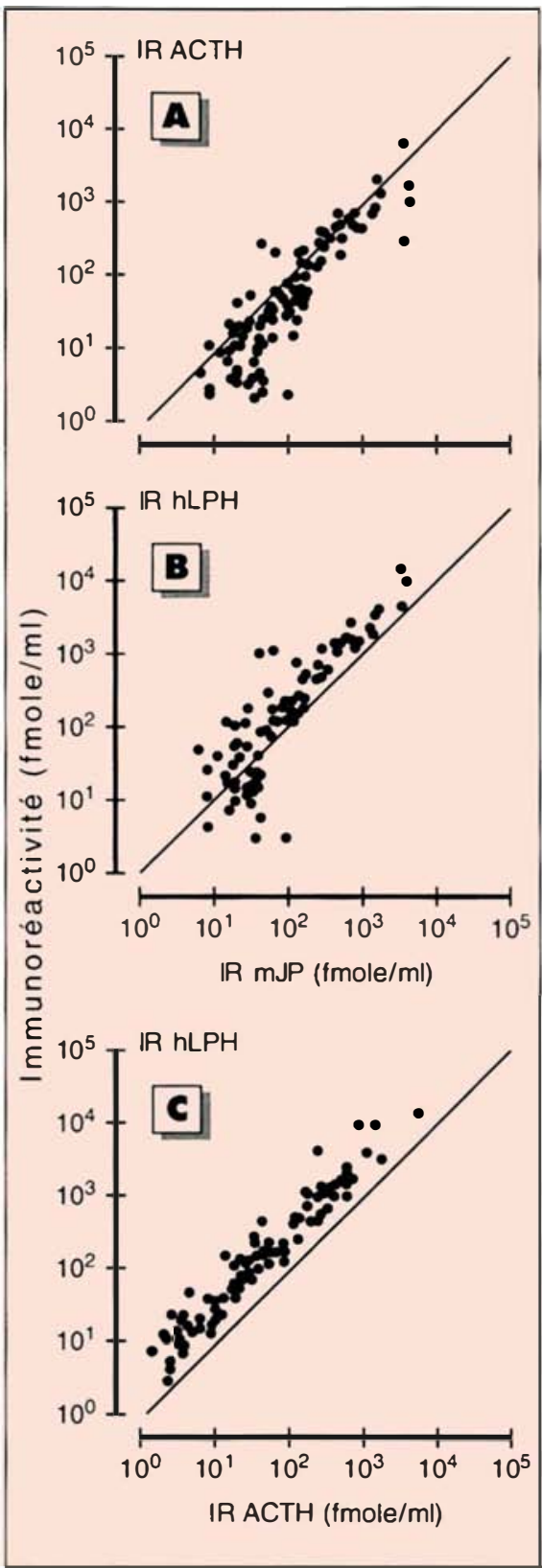

Figure 2. Corrélations des immunoréactivités plasmatiques ACTH, LPH et JP chez 115 patients. La droite de régression théorique de 1 mole pour 1 mole est représentée dans les trois cas. 


\section{RÉFÉRENCES}

12. Lacaze-Masmonteil $T$, De Keyzer $Y$, Luton JP, Kahn A, Bertagna X. Characterization of proopiomelanocortin transcripts in human non-pituitary tissues. Proc Natl Acad Sci USA 1987 ; 84 : 7261-5.

13. De Bold CR, Nicholson WE, Orth DN. Immunoreactive proopiomelanocortin (POMC) peptides and POMC-like messenger ribonucleic acid are present in many rat nonpituitary tissues. Endocrinology 1988 ; 122 : 2648-57.

14. Jingami $H$, Nakanishi $S$, Imura $H$, Numa S. Tissue distribution of messenger RNAs coding for opioid peptide precursors and related RNA. Eur J Biochem 1984; 142 : $441-7$.

15. Jeannotte L, Burbach JPH, Drouin J. Unusual proopiomelanocortin ribonucleic acids in extrapituitary tissues : intronless transcripts in testes and long poly (A)+ tails in hypothalamus. Mol Endocrinol 1987 ; 1 : 749-57.

16. Eipper BA, Mains RE. Structure and biosynthesis of pro-adreno-corticotropin/endorphin and related peptides. Endocr Rev 1980 ; 1: 1-27.

17. Seidah NG, Gaspar L, Mion P, Marcinkiewicz $\mathrm{M}$, Mbikay $\mathrm{M}$, Chrétien $\mathrm{M}$. cDNA sequence of two distinct pituitary proteins homologous to KEX 2 and furin gene products : tissue-specific mRNAs encoding candidates for pro-hormone processing proteinases. DNA Cell Biol 1990; 9 : 415-24.

18. Smeekens SP, Steiner DF. Identification of a human insulinoma cDNA encoding a novel mammalian protein structurally related to the yeast dibasic processing protease KEX 2. J Biol Chem 1990; 265 : 2987-3000.

19. Seidah NG, Marcinkiewicz M, Benjannet $\mathrm{S}$ et al. Cloning and primary sequence of a mouse candidate prohormone convertase PC1 homologous to PC2, furin and KEX 2 ; distinct chromosomal localization and messenger RNA distribution in brain and pituitary compared to PC2. Mol Endocrinol 1991, 5 : 111-22.

*** Ce dosage biologique s'effectue sur des coupes de cortico-surrénales. Il utilise la propriété qu'à l'ACTH de dépléter la cellule surrénalienne en ascorbate et donc de diminuer son potentiel réducteur. Des techniques de cytochimie quantitative par microdensitométrie permettent d'apprécier l'état d'oxydoréduction de la cel- utilisés pour mesurer l'ACTH, notamment le dosage par radio-récepteurs et le dosage redox*** qui présentent l'avantage de mesurer l'ACTH bioactive et, en ce qui concerne le dosage redox, d'être d'une très, très grande sensibilité (environ 100 fois supérieure à celle d'un RIA classique).

Cependant l'ACTH n'est que l'un des signaux émis par la cellule corticotrope. Qu'en est-il des autres peptides dérivés de la POMC et comment les utiliser de façon pertinente en clinique?

\section{- Peptides circulants non ACTH} de la POMC

- Taux plasmatiques des différents peptides dérivés de la POMC

Tous les peptides de la POMC ont été mesurés par RIA dans le sang chez l'homme. On peut s'attendre que la mesure de l'un quelconque d'entre eux offre exactement le même indice de l'activité sécrétoire des cellules corticotropes antéhypophysaires. En première approximation, les immunoréactivités dérivées de la POMC ont été comparées entre elles par un choix judicieux des cohortes de patients balayant de larges variations dans le niveau de la fonction corticotrope.

A titre d'exemple, la figure 2, p. 921 indique les corrélations des immunoréactivités plasmatiques $\mathrm{JP}, \mathrm{LPH}$ et ACTH : les bonnes corrélations obte- nues avec un rapport molaire proche de 1 indiquent, en première estimation, que les peptides satellites dérivés de la POMC sont effectivement sécrétés avec l'ACTH et, de ce fait, apportent le même type d'informations.

- Cas particulier des LPH et du JP Parmi ces satellites, les LPH ont été étudiées de manière extensive, car elles présentent un certain nombre d'avantages leur conférant un intérêt tout particulier dans l'exploration de la fonction corticotrope en clinique. Les LPH sont beaucoup plus résistantes à la protéolyse, et donc plus stables dans le plasma que l'ACTH. Les prélèvements sanguins peuvent être conservés pendant 24 heures à la température de la pièce sans dégradation de ces peptides [35]. Par ailleurs, la partie $\mathrm{N}$-ter commune de la $\gamma$ et de la $\beta$-LPH est très immunogène, rendant aisée l'obtention d'anticorps. Les RIA des LPH détectent donc, en réalité, les deux LPH : $\beta$ et $\gamma$. C'est par abus de langage qu'on parle du dosage de la LPH plasmatique. Pour ces deux raisons, et aussi parce que les LPH ont été les premiers peptides non ACTH de la POMC disponibles, leur étude a été plus étendue et leur utilisation est aujourd'hui la plus fréquente. Celleci reste limitée, toutefois, car peu d'équipes possèdent les peptides humains, uniquement obtenus jusqu'ici par purification. Les LPH

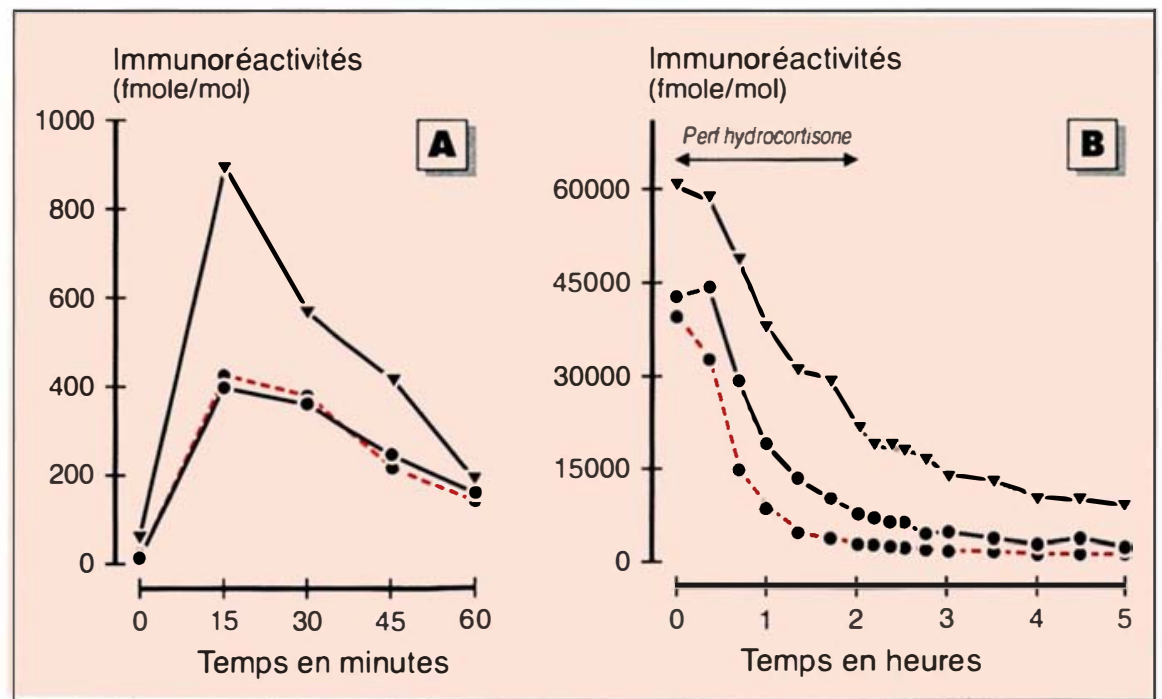

Figure 3. Variations parallèles des immunoréactivités plasmatiques ACTH, LPH et JP lors de tests dynamiques. Stimulation de la fonction corticotrope lors du test au CRF (corticotropin releasing factor) dans une maladie de Cushing $(A)$; freination de la fonction corticotrope par perfusion de cortisol dans

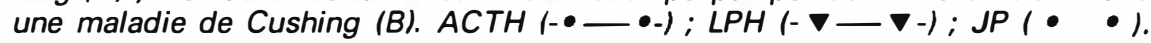


Tout récemment, le dosage plasmatique du JP humain a été développé sur un principe original. Anticipant sur l'idée que ce peptide humain devait être amidé en C-ter, nous avons vérifié qu'il croisait parfaitement dans un RIA dirigé contre la même séquence C-ter du peptide murin, elle aussi amidée [36]. Le RIA, très sensible s'est révélé remarquablement utile, en particulier en raison d'une durée d'incubation très courte (24 heures).

Des études extensives ont été menées, qui montrent que ACTH et LPH (et plus récemment le $\mathrm{JP}$ ) ont des variations plasmatiques parallèles, qu'il s'agisse de rythme circadien normal, de réponses aux différents tests de stimulation (arginine-vasopressine, corticotropin releasing factor, métopirone, hypoglycémie insulinique) ou de freination (dexaméthasone) pharmacologiques. A titre d'exemple (figure 3) sont montrées les variations parallèles des immunoréactivités ACTH, LPH et JP sous l'effet freinateur de cortisol (ou hydrocortisone) ou stimulant du CRF.

\section{Les fragments de la POMC en pathologie humaine}

Certaines situations pathologiques modifient les rapports normaux entre les différents fragments de la POMC dans le sang circulant. Ces phénomènes résultent soit d'un désordre retentissant sur le métabolisme périphérique de certains peptides, soit d'une anomalie acquise de la maturation de la POMC, surtout le fait de certaines tumeurs. La seule évaluation de l'ACTH circulante peut alors être insuffisante.

\section{- L'insuffisance rénale}

Contrairement à l'ACTH, les LPH ont un catabolisme essentiellement rénal. En cas d'insuffisance rénale même modérée, la LPH plasmatique s'élève de façon significative. Ce phénomène se retrouve exacerbé chez certains hémodialysés qui peuvent présenter une véritable pigmentation addisonienne. En revanche, l'ACTH et donc le cortisol restent à des taux strictement normaux. Les études de freination par l'administration aiguë ou prolongée de dexaméthasone ont bien montré la suppression strictement normale de l'ACTH et du cortisol alors que la LPH plasmatique décroît sur une pente d'élimination beaucoup plus lente.

Un autre peptide de la POMC, le $\mathrm{JP}$, est également augmenté de façon tout à fait discordante dans cette situation. Les rapports LPH/ACTH ou JP/ACTH sont donc tous les deux anormaux. L'existence d'une mélanodermie, d'un amaigrissement, de troubles digestifs, d'une altération de l'état général, de modifications ioniques peut évoquer à tort le dia-

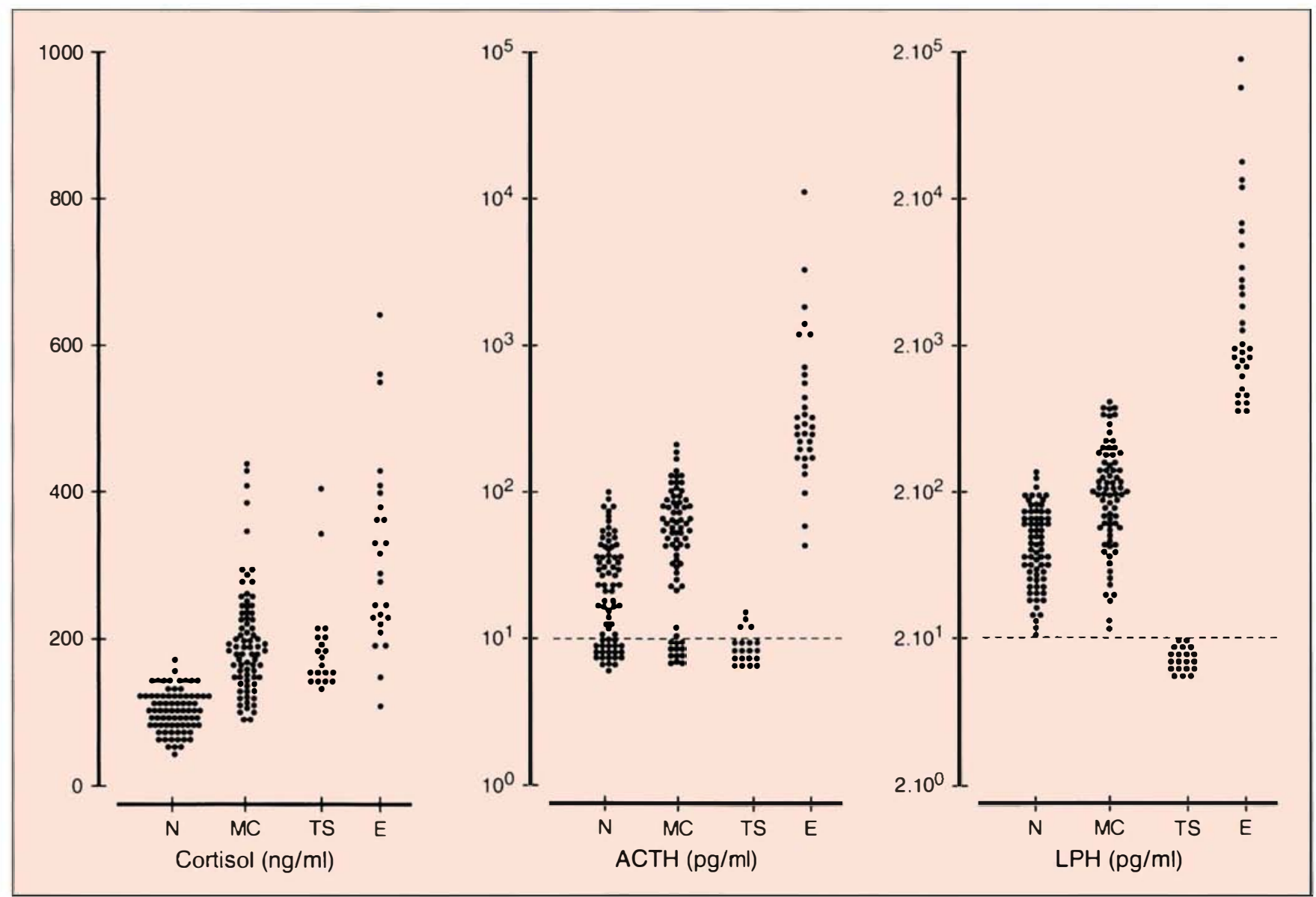

Figure 4. Concentrations de cortisol, ACTH et LPH dans un prélèvement plasmatique de base, le matin, chez des sujets normaux $(N)$ et des patients atteints de syndrome de Cushing par maladie de Cushing (MC), tumeur surrénalienne (TS) et syndrome de sécrétion ectopique (E). 


\section{RÉFÉRENCES}

20. Benjannet $\mathrm{S}$, Rondeau $\mathrm{N}$, Day $\mathrm{R}$, Chrétien M, Seidah NG. PC1 and PC2 are proprotein convertases capable of cleaving proopiomelanocortin at distinct pairs of basic residues. Proc Natl Acad Sci USA 1991 ; 88 : 3564-8.

21. Seidah NG, Chrétien M. Complete amino acid sequence of a human pituitary glycopeptide : an important maturation product of proopiomelanocortin. Proc Natl Acad Sci USA 1981; 78 : 4236-40.

22. Mains RE, Eipper BA. Phosphorylation of rat and human adrenocorticotropin related peptides : physiological regulation and studies of secretion. Endocrinology 1983 ; 112 : 1986-5.

23. Seidah NG, Rochemont J, Hamelin J, Benjannet $\mathrm{S}$, Chrétien $\mathrm{M}$. The missing fragment of the prosequence of human proopiomelanocortin: sequence and evidence for C-terminal amidation. Biochem Biophys Res Commun 1991; 102 : 710-6.

24. Glembotski CC. Acetylation of alpha $\mathrm{MSH}$ and $\beta$-endorphin in the rat intermediate pituitary. J Biol Chem 1982 ; 257 : 10493-500.

25. Smyth DG, Massey DE, Zakarian S, Finnie MDA. Endorphins are stored in biologically active and inactive forms : isolation of $2 \mathrm{~N}$ acetyl peptides. Nature 1979 ; $279: 252-4$

26. Rudman DI, Chawla RK, Hollins BM $\mathrm{N}$, O-diacetyl serine $\alpha$-melanocyte stimulating hormone, a naturally occurring melanotropic peptide. J Biol Chem 1979; 254 : 10102-8.

27. Pedersen RC, Brownie AC, Ling $\mathrm{N}$, Pro-adrenocorticotropin/endorphin derived peptides : coordinate action on adrenal steroidogenesis. Science 1980 ; 208 : 1044-5.

28. Seidah N, Rochemont J. Hamelin J. Lis M, Chrétien M. Primary structure of the major human pituitary proopiomelanocortin NH2-terminal glycopeptide. Evidence for an aldosterone-stimulating activity. $J$ Biol Chem 1981; 256 : 7977-84.

29. Pham Huu Trung MT, De Smitter N, Boggio A, Bertagna X, Girard F. Responses of isolated Guinea-pig adrenal cells to ACTH and proopiocortin derived peptides. gnostic de maladie d'Addison chez ces patients. Les mesures de l'ACTH et du cortisol rétablissent le diagnostic.

- POMC et syndromes de Cushing L'hypercortisolisme chronique, ou syndrome de Cushing, survient dans deux circonstances. L'hypersécrétion des glucocorticoïdes peut être primitivement surrénalienne [37]. Plus fréquemment, l'hypercortisolisme est secondaire à une hypersécrétion corticotrope qui peut elle-même avoir deux origines. Le plus souvent, elle est hypophysaire ou ectopique ; il s'agit de la maladie de Cushing [38].
Plus rarement, elle est ectopique par une tumeur non hypophysaire [39]. L'évaluation des peptides dérivés de l'ACTH et des LPH, participe au diagnostic étiologique du syndrome de Cushing.

Hypercortisolisme par tumeur surrénalienne La sécrétion corticotrope est mise au repos et les taux circulants d'ACTH et de LPH sont effondrés (figure 4, p. 923) et non réactivables par les tests de stimulation.

Hypercortisolisme par hypersécrétion corticotrope eutopique (maladie de Cushing)

L'évaluation des taux circulants d'ACTH et de LPH et de leur évola POMC, et plus particulièrment de

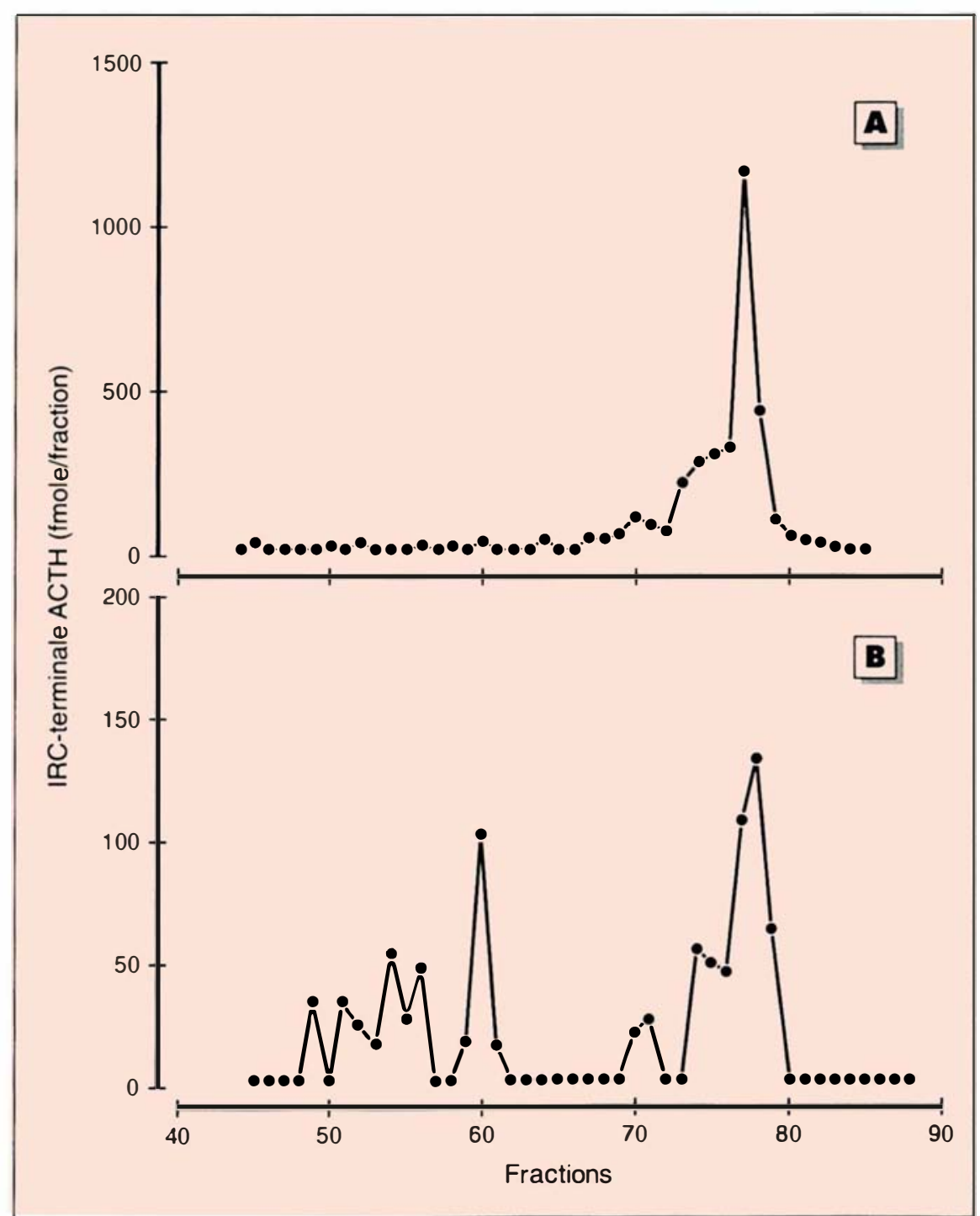

Figure 5. Séparation par HPLC de l'immunoréactivité plasmatique ACTH C-terminale chez un patient avec maladie de Cushing $(A)$ et un patient avec le syndrome de sécrétion ectopique d'ACTH (B). Les fractions 74-80 correspondent aux peptides de type $\mathrm{ACTH}_{1-39}$; les fractions 49-61, aux peptides de type CLIP. 
lution sous divers stimuli va le plus souvent permettre de préciser leur origine hypophysaire: les valeurs d'ACTH ne sont que modérément élevées (figure 4), mais cependant de façon inadaptée à l'état d'hypercortisolisme. L'ACTH et les LPH sont sécrétées de façon équimolaire de telle sorte que le rapport de leur concentration, exprimée en $\mathrm{pg} / \mathrm{ml}$, est voisin de 3, et en tout cas inférieur à 5 . En outre, il persiste une régulation de la sécrétion corticotrope sans autonomie sécrétoire dont rend compte la capacité qu'ont l'argininevasopressine, le corticotropin releasing factor et la métopirone de stimuler les sécrétions parallèles d'ACTH et de LHP et les glucocorticoïdes de les freiner partiellement.

Hypercortisolisme par hypersécrétion corticotrope d'origine ectopique

Les taux plasmatiques d'ACTH et de $\mathrm{LPH}$ sont en général très augmentés et presque toujours supérieurs à ceux observés dans la maladie de Cushing (figure 4). Cette sécrétion ectopique est autonome, ce dont rend compte l'absence de variations aux différents tests de stimulation ou de freination de la sécrétion corticotrope. On constate très fréquemment une dissociation des valeurs d'ACTH et de LPH. Le rapport habituel de leurs concentrations est déséquilibré en faveur de ces dernières et se trouve très nettement supérieur à 5 .

La dissociation du rapport LPH/ACTH dans le sang de ces patients est, en réalité, le témoin d'une maturation inhabituelle de la POMC dans la plupart des tumeurs non hypophysaires responsables d'une sécrétion ectopique d'ACTH. Les études faites directement sur de telles tumeurs montrent que certains sites de protéolyse du précurseur, normalement préservés dans l'antéhypophyse, que celle-ci soit normale ou tumorale, sont activés dans ces tumeurs non hypophysaires. Le fragment anormal le plus souvent repéré est le CLIP [40] ; pour chaque molécule de CLIP formée, une molécule d'ACTH est perdue pour le dosage RIA ou IRMA, d'où l'augmentation du rapport LPH/ACTH qui tient en fait à la diminution du dénominateur. L'observation que le rapport JP/ACTH est lui aussi augmenté, parallèlement au rapport LPH/ $m / s n^{\circ} 9$, vol. 7, novembre 91

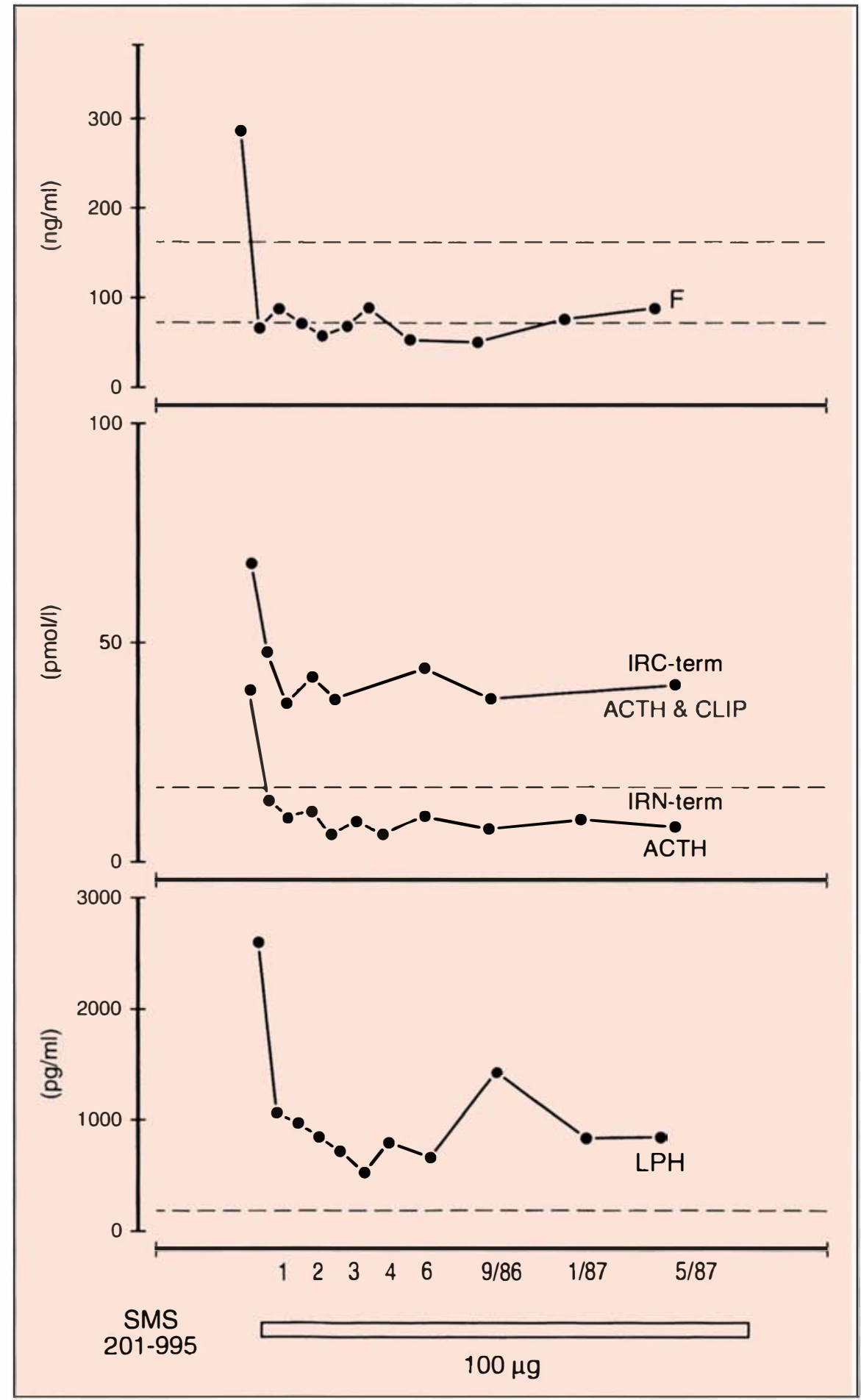

Figure 6. Effet d'un traitement par l'octréotide (SMS 201-995) sur les variations plasmatiques d'ACTH ${ }_{1-39}$ CLIP, cortisol (F) et $L P H$ chez un patient avec le syndrome de sécrétion ectopique d'ACTH. IRC-term : immunoréactivité C-terminale; IRN-term : immunoréactivité $N$-terminale. 


\section{RÉFÉRENCES}

30. Penhoat A, Sanchez P, Jaillard C, Langlois D, Begeot M, Saez JM. Human proopiomelanocortin (79-76), a proposed cortical-androgen stimulating hormone does not affect steroidogenesis in cultured human adult adrenal cells. J Clin Endocrinol Metab $1991 ; 72$ : 23-6.

31. Williams WC, Island D, Oldfield RAA, Grant W. Blood corticotropin (ACTH) levels in Cushing's disease. J Clin Endocrinol Metab 1961; 21 : 426-32.

32. Yalow RS, Glick SM, Roth J, Berson SA. Radio-immunoassay of human plasma ACTH. J Clin Endocrinol 1964 ; 24 : 1219.

33. Lowry PJ, Linton EA, Hodgkinson SC. Analysis of peptide hormones of the hypothalamic pituitary adrenal axis using " twosite "immunoradiometric assays. Horm Res $1989 ; 32: 25-9$.

34. Raff H, Findling JW, Wong J. Short loop adrenocorticotropin (ACTH) feedack after ACTH-(1-24) injection in man is an artifact of the immunoradiometric assay. $J$ Clin Endocrinol Metab 1989; 69 : 678-80.

35. Kuhn JM, Seurin D, Bertagna X, Hadjiat R, Thieblot P, Girard F. Les lipotropines $(\beta$ - et $\gamma$-LPH) marqueurs fiables de la fonction corticotrope. Ann Endocrinol $1984 ; 45$ : 369-74.

36. Bertagna $\mathrm{X}$, Camus $\mathrm{F}$, Lenne $\mathrm{F}$, Girard F, Luton JP. Human joining peptide : a proopiomelanocortin product secreted as a homodimer. Mol Endocrinol 1988 ; 2 : 1108-14.

37. Gold EM. The Cushing syndromes : changing views of diagnosis and treatment. Ann Intern Med 1979 ; 90 : 829-44.

38. Cushing H. The basophil adenomas of the pituitary body and their clinical manifestation. Bull Johns Hopkins Hosp 1932 ; 50 : 137-95.

39. Meador CK, Liddle GW, Island DP, et al. Cause of Cushing's syndrome in patients with tumors arising from "nonendocrine " tissue. I Clin Endocrinol Metab $1962 ; 22$ : 6893-703.

40. Vieau D, Massias JF, Girard F, Lutton JP, Bertagna $\mathrm{X}$. Corticotropin-like intermediary lobe peptide as a marker of alternative proopiomelanocortin processing in ACTH-producing non-pituitary tumours. Clin Endocrinol 1989 ; 31 : 691-700.

**** L'octréotide (ou SMS 201-995) est un analogue synthétique de la somatostatine. Sa durée d'action plus prolongée permet son utilisation pharmacologique en clinique. Il s'est avéré particulièrement efficace pour freiner les sécrétions hormonales des tumeurs endocrines du pancréas, y compris certains cas de sécré-
ACTH, renforce encore cette interprétation.

Le CLIP a pu être mis en évidence dans le plasma de ces patients. L'approche a été tout d'abord indirecte, en utilisant des dosages de spécificité différente (détermination de l'immunoréactivité C-ter évaluant conjointement $\mathrm{ACTH}_{1-39}$ et CLIP, d'une part, et détermination de l'immunoréactivité $\mathrm{N}$-ter évaluant uniquement l'ACTH ${ }_{1.39}$, d'autre part). Il existe une bonne corrélation entre les rapports d'immunoréactivités C-ter/N-ter et LPH/ACTH. Le CLIP a, par la suite, pu être mis en évidence directement dans le plasma de ces sujets par des techniques d'HPLC (figure 5, p. 924).

Parfois, la POMC subit, dans des tumeurs à sécrétion ectopique, une maturation moins poussée puisqu'elle a pu être mise en évidence intacte. Dans les deux cas, peu ou pas de maturation et maturation excessive, le dosage IRMA de l'ACTH ne reconnaissant que l'ACTH $\mathrm{H}_{1-39}$ peut présenter des dangers en sousestimant l'immunoréactivité globalement dérivée de la POMC.

C'est dans ces situations assez rares, mais néanmoins classiques, que certains RIA, et surtout les IRMA, sont pris en défaut par excès... de spécificité. Ce type de tumeurs - parfois véritables "clipomes " - sécrètent peu d'ACTH ${ }_{1.39}$, beaucoup de CLIP, beaucoup de LPH... Non seulement le diagnostic mais aussi le suivi thérapeutique réclament la mesure de ces peptides non ACTH pour apprécier l'efficacité de la thérapeutique. L'exemple de la figure 6 , p. 925 montre le cas d'un patient traité par l'octréotide**** (SMS 201-995) pour un cancer pancréatique endocrine : alors que l'ACTH et le cortisal sont normalisés, l'évaluation de la LPH et du CLIP montre la persistance de taux anormalement élevés - sans conséquences cliniques puisque ces peptides sont inactifs sur les surrénales - mais traduisant la persistance d'un phénomène tumoral pathologique imparfaitement contrôlé. Les peptides non ACTH de la POMC, et particulièrement les LPH, offrent une alternative précieuse pour l'évaluation de la fonction corticotrope. Au-delà des affections tumorales envisagées plus tôt, ils sont éga- lement indispensables dans quelques situations, assez exceptionnelles, où le dosage de l'ACTH est impossible: présence d'anticorps anti-ACTH, traitement par le synacthène $\left(\mathrm{ACTH}_{1-24}\right.$ synthétique), artefacts du plasma, hémolyse. La stabilité des LPH dans le sang permet d'envisager leur mesure dans des échantillons obtenus par des systèmes de prélèvements continus sur 24 heures, un objectif strictement original qui, pour la première fois, pourrait évaluer, en parallèle, la fonction corticotrope et la réponse surrénalienne sur la durée

\section{Summary}

Proopiomelanocortin and its peptide-fragments : clinical aspects

Proopiomelanocortin (or POMC), the polypeptide precursor to ACTH, also generates a series of other peptide-fragments during the maturation process which takes place in the normal pituitary and in various $\mathrm{POMC}$-producing pituitary -or non-pituitarytumors. Thus $\beta$-endorphin-, the lipotropins $(\beta$ - and $\gamma-$ ), the Joining Peptide, the $\mathrm{N}$-terminal fragment all circulate in blood and are valid alternate markers of the overall pituitary corticotropic function. An abnormal maturation of POMC occurs in some nonpituitary tumors releasing aberrant peptide fragments such as $\beta \mathrm{MSH}_{5.22}$ and CLIP (or $\mathrm{ACTH}_{18.39}^{j .22}$ ). This latter peptide will escape the detection by highly specific ACTH radioimmunoassays and the commercially available two-site immunoradiometric assays.

\section{TIRÉS A PART}

D. Vieau. 